\title{
A Hybrid Medium Voltage Multilevel Converter with Parallel Voltage-Source Active Filter
}

\author{
Daniel Bernet, Lukas Stefanski and Marc Hiller \\ Karlsruhe Institute of Technology, \\ Institute of Electrical Engineering (ETI) \\ Kaiserstr. 12, 76131 Karlsruhe, Germany \\ daniel.bernet@kit.edu
}

\begin{abstract}
In consequence of high semiconductor losses, gridconnected medium voltage power converters are typically operated at switching frequencies of several hundred Hertz per switch position. Therefore, conventional converter systems require bulky and expensive LCL-filters in order to meet the harmonic limits given by the grid code. It is only possible to reduce the $\mathrm{LCL}-$ filter costs by semiconductor current derating and operation at increased switching frequencies, leading to a reduced utilization and efficiency of the converter system. To overcome these disadvantages of conventional converter systems, the presented hybrid converter uses a parallel voltagesource active output filter and thus allows a significant reduction of the passive component demand. An excellent harmonic performance is achieved for the operation with small passive filter components, revealing the potential for increasing the power density and efficiency of high power medium voltage converters. As a result, significant reductions of the filter losses and passive components as well as an increased utilization are achieved compared to a reference LCL-filter based converter system.
\end{abstract}

Index Terms-Hybrid converter systems, active filter, medium voltage converter, multilevel converter, LCL-filter

\section{INTRODUCTION}

Emerging applications such as Solid-State-Transformers (SST) for grid couplings, Medium-Voltage-DC-TransmissionSystems (MVDC), Flexible-AC-Transmission-Systems (FACTS) and medium voltage electric drives use gridconnected medium voltage converters. In [1], voltage source converters (VSC) for industrial medium voltage applications are classified and compared. As a result of competitive costs, high robustness and low complexity, half of the commercially available converter solutions (e.g. by ABB, SIEMENS, TMEIC) in the lower medium voltage range from $2.3 \mathrm{kV}$ to $4.16 \mathrm{kV}$ use the $3 \mathrm{~L}-$ Neutral Point Clamped (NPC) or the 3L-Active Neutral Point Clamped (ANPC) converter [2, 3], respectively. In consequence of significant semiconductor losses resulting from high blocking voltages and load currents, the switching frequency of the 3L-NPC is typically limited to below $600 \mathrm{~Hz}$ per switch. Therefore,

The authors would like to thank the German Federal Ministry of Education and Research for funding this research within the ENSURE project under the grant FKZ 03SFK1A.

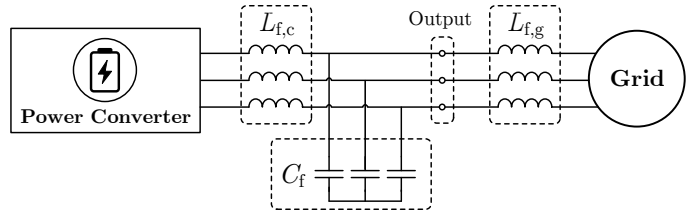

Fig. 1: Conventional LCL-filter based grid-connected medium voltage converter system

conventional converter systems use passive LCL-filters composed of bulky inductors and capacitors as shown in Fig. 1 for meeting the harmonic limits (e.g. given by IEEE Standard 519 [4]). In order to limit the resulting LCL-filter costs that often surpass the costs of the converter, the LCL-filter inductances and capacitances need to be reduced. Beyond optimized modulation schemes based on Optimized Pulse Patterns (OPP), e.g. investigated in [5] for medium voltage drives, this can only be achieved by derating the semiconductor current and increasing the switching frequency, leading to a reduced utilization and efficiency of the converter system. Further important disadvantages of LCL-filter based converter systems are substantial additional losses, reduced dynamic performance, frequency dependency and susceptibility to oscillation, requiring significant and lossy damping.

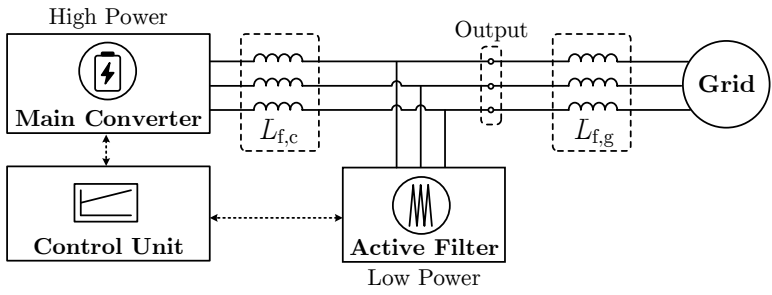

Fig. 2: Hybrid medium voltage converter combining a high power main converter and a low power active filter

In order to overcome the aforementioned disadvantages of conventional LCL-filter based converter systems, we introduced the combination of a high power main converter (MC) and a low power parallel voltage-source active filter (AF) for low voltage applications in [6]. Therein, the implementation of a coupled control scheme on a single 
control unit according to Fig. 2 allows the mitigation of the main converter switching frequency current ripple and thereby the replacement of the passive LCL-filter. In a further publication, we use the hybrid converter concept to design a hybrid medium voltage multilevel converter and to investigate the impact on important converter characteristics such as passive component demand and filter losses. It is shown that replacing the filter capacitors by a low power active filter increases the installed semiconductor power by $11 \%$, but eliminates important disadvantages of passive LCL-filters. As a result, the total filter inductance and the energy stored therein is reduced by over $60 \%$, significantly reducing the filter costs. Furthermore, since the resonant circuit is eliminated by the active output filter, no damping circuit is required for the hybrid converter.

This contribution focuses on the achievable utilization and efficiency of the hybrid converter compared to LCLfilter based high power medium voltage converters. The proposed hybrid converter topology and the implemented control scheme including the active filter energy control is presented in Section II. In Section III, the simulation results illustrate the harmonic performance of the hybrid converter under reasonable medium voltage grid conditions. In order to evaluate the potential for grid applications, important converter characteristics of the hybrid converter and conventional converter systems are investigated in Section IV. Therein, the achievable converter utilization and efficiency are compared over the operating range.

\section{Converter Topology and Control}

Fig. 3 shows the proposed hybrid medium voltage multilevel converter. To achieve competitive costs, high robustness and high power density, a conventional 3L-NPC based on silicon insulated-gate bipolar transistors ( $\mathrm{Si}$-IGBTs) is used as main converter. The topology used as active filter is composed of cascaded full-bridge cells in star configuration and is referred to as a Cascaded H-Bridge Active Filter (CHB AF). The design and control of CHB converters for STATCOM applications has been investigated in previous works [7-9]. They are commercially available e.g. as SVC PLUS by SIEMENS [10] or SVC Light by ABв [11].

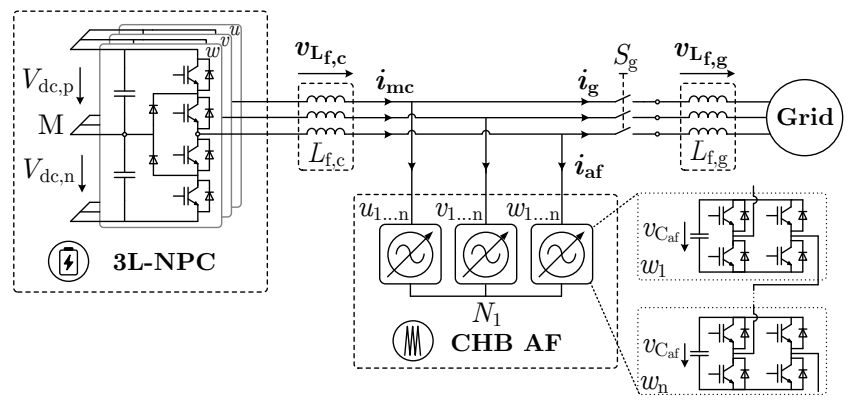

Fig. 3: Proposed hybrid medium voltage multilevel converter composed of a 3L-NPC as main converter and a CHB Active Filter (AF) in star configuration

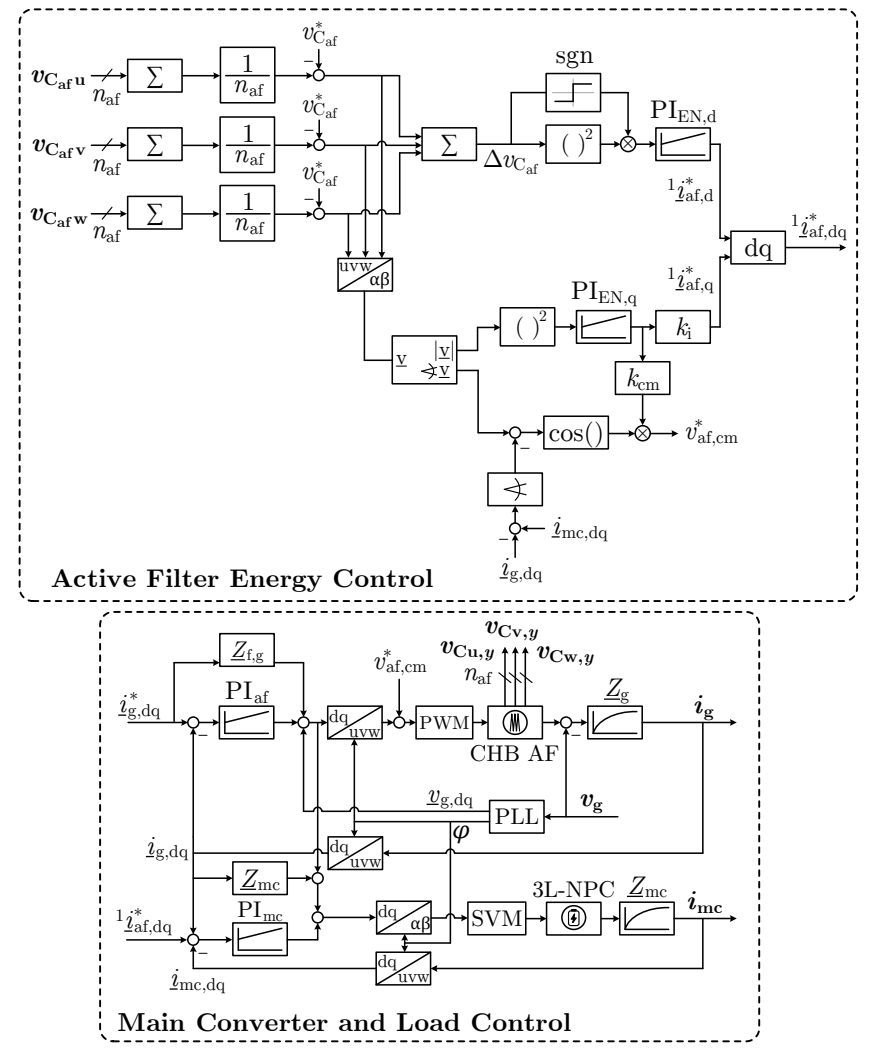

Fig. 4: Control scheme of the hybrid converter system: active filter energy control (top), main converter and load control (bottom)

The main converter supplies the active power of the hybrid converter system. In contrast, the active filter compensates the main converter current ripple and is ideally not exchanging active or fundamental reactive power, allowing a low CHB AF power rating. Compared to full power multilevel converters with comparable characteristics, the decoupling of high and low power components within the hybrid converter system offers important advantages such as high power density and efficiency. At the same time, the use of a conventional IGBT-based high power converter leads to a high robustness and competitive costs.

This contribution presents the hybrid converter control in Fig. 4, including the active filter energy control and the main converter and load control as introduced in [6]. The design of the energy control is based on $[12,13]$ and adapted to the star-configured Cascaded H-Bridge Converter Active Filter with $n_{\text {af }}$ cascaded full-bridge cells per phase. According to (1), the stored energy $W_{\mathrm{C}_{\mathrm{af}} x, y}$ can be determined using the cell capacitance $C_{\mathrm{af}}$ and the measured cell voltage $v_{\mathrm{C}_{\mathrm{af}} x, y}$ with $x=\{u, v, w\}$ and $y=\left\{k \in \mathbb{N} \mid 1 \leq k \leq n_{\mathrm{af}}\right\}$.

$$
W_{\mathrm{C}_{\mathrm{af}} x, y}=\frac{1}{2} C_{\mathrm{af}} v_{\mathrm{C}_{\mathrm{af}} x, y}^{2}
$$

During operation, a total energy deviation as well as an unsymmetrical energy distribution between the three phases 
of the CHB AF can occur. A total energy deviation is characterized by

$$
\left|\Delta v_{\mathrm{C}_{\mathrm{af}}}\right|=\left|\bar{v}_{\mathrm{C}_{\mathrm{af}}}-v_{\mathrm{C}_{\mathrm{af}}}^{*}\right|>0
$$

with the reference cell voltage $v_{\mathrm{C}_{\mathrm{af}}}^{*}$ and the mean cell voltage

$$
\bar{v}_{\mathrm{C}_{\mathrm{af}}}=\frac{1}{3 n_{\mathrm{af}}} \sum_{y=1}^{n_{\mathrm{af}}}\left(v_{\mathrm{C}_{\mathrm{af}} \mathrm{u}, y}+v_{\mathrm{C}_{\mathrm{af}} \mathrm{v}, y}+v_{\mathrm{C}_{\mathrm{af}} \mathrm{w}, y}\right) .
$$

For $\Delta v_{\mathrm{C}_{\mathrm{af}}}<0$, the total energy is below the reference energy and a positive active power exchange is required. Likewise, the total energy is above its reference value for $\Delta v_{\mathrm{C}_{\mathrm{af}}}>0$ and a negative active power exchange is required. If the active filter exchanges no active power with the grid or the main converter, semiconductor and capacitor losses lead to a continuous decrease of the stored energy and thus require a small positive active power exchange during operation. In $d q$-coordinates, the fundamental current ${ }^{1} i_{\mathrm{af}, \mathrm{d}}$ corresponds to an active current in phase with the output voltage and therefore is used for total energy control.

An unsymmetrical energy distribution can be caused by unsymmetrical main converter current ripples that correspond to the load current of the active filter. In addition, unsymmetrical grid conditions lead to an unequal power exchange of the active filter phases and thus can cause an unsymmetrical energy distribution as well. Hence, a Clarke-Transformation of the three-phase voltage deviation is proposed in [12]. The phase angle of the resulting space vector relates to the distribution of the energy deviation among the three phases, whereas the amplitude indicates the deviation intensity. The use of an active filter fundamental common mode (CM) voltage $v_{\mathrm{af}, \mathrm{cm}}=\hat{v}_{\mathrm{af}, \mathrm{cm}} \cos \left(\omega_{\mathrm{g}} t+\varphi_{\mathrm{af}, \mathrm{cm}}\right)$ (where $\omega_{\mathrm{g}}=2 \pi f_{\mathrm{g}}$ is the grid angular frequency and $\varphi_{\mathrm{af}, \mathrm{cm}}$ the phase shift) and fundamental current ${ }^{1} \underline{i}_{\mathrm{af}, \alpha \beta}={ }^{1} \hat{i}_{\mathrm{af}} e^{j\left(\omega_{\mathrm{g}} t+\varphi_{\mathrm{af}, \mathrm{i}}\right)}$ (where $j$ is the imaginary unit and $\varphi_{\mathrm{af}, \mathrm{i}}$ the phase shift) leads to the additional power component

$$
\underline{p}_{\mathrm{cm}, \alpha \beta}=\frac{\hat{v}_{\mathrm{af}, \mathrm{cm}}{ }^{1} \hat{i}_{\mathrm{af}}}{2}\left(\underline{\tilde{a}}_{\mathrm{cm}, \alpha \beta}+\underline{\bar{a}}_{\mathrm{cm}, \alpha \beta}\right) \text {. }
$$

In (4), $\underline{\tilde{a}}_{\mathrm{cm}, \alpha \beta}=e^{j\left(2 \omega_{\mathrm{g}} t+\varphi_{\mathrm{af}, \mathrm{i}}+\varphi_{\mathrm{af}, \mathrm{cm}}\right)}$ is an alternating and $\underline{\bar{c}}_{\mathrm{cm}, \alpha \beta}=e^{j\left(\varphi_{\mathrm{af}, \mathrm{i}}-\varphi_{\mathrm{af}, \mathrm{cm}}\right)}$ a DC component. To ensure a limited energy pulsation in the active filter phase-legs, the alternating component needs to be considered for the converter design. However, the DC component can be used to achieve an energy transfer between the active filter phases and thereby eliminates the unsymmetrical energy distribution. As shown in Fig. 4, the parameters $k_{\mathrm{i}}$ and $k_{\mathrm{cm}}$ are used to impact the trade-off between the energy balancing related use of converter current and common mode voltage. The arbitrary phase shift of the common mode voltage allows the use of active and reactive fundamental currents. Since active current is required for the total energy control, an operation with minimal reactive current is reasonable in order to reduce the overall current load.

\section{Simulation Results}

Simulation results are carried out for the grid-connected hybrid converter system shown in Fig. 5. It is composed of the 3L-NPC main converter, the CHB Active Filter, a main converter-side filter inductance and the three-phase medium voltage transformer. The converter parameters are given in Table I. Conventional Si-IGBT modules FZ1200R45HL3 (INFINEON) with a collector-emitter voltage of $V_{\mathrm{CES}}=4500 \mathrm{~V}$ and a continuous DC collector current of $I_{\mathrm{C}, \text { nom }}=1200 \mathrm{~A}$ are used in the $3 \mathrm{~L}-\mathrm{NPC}$ main converter. The active filter design is based on the Si-IGBT module FF150R12RT4 (INFINEON) with a collector-emitter voltage of $V_{\mathrm{CES}}=1200 \mathrm{~V}$ and a continuous DC collector current of $I_{\mathrm{C}, \text { nom }}=150 \mathrm{~A}$. Taking into account the transformer leakage inductance of $L_{\mathrm{T} \sigma}=0.42 \mathrm{mH}$ resulting from the short-circuit voltage, the multilevel output voltage and high effective switching frequency of the CHB AF allow a grid code compliant operation without additional grid-side filter inductance. According to common grid codes, the permitted grid voltage deviation is $\pm 10 \%$ of the rated grid voltage. The power factor range $\cos (\varphi) \geq 0.9$ is considered for inductive and capacitive loads. As a result, the transformer turns ratio is determined to

$$
u_{\mathrm{T}, \mathrm{af}}=\frac{v_{\mathrm{T}}}{v_{\mathrm{PCC}}}=0.91,
$$

where $v_{\mathrm{T}}$ is the primary transformer voltage and $v_{\mathrm{PCC}}$ the grid voltage at the point of common coupling (PCC). The DC-link voltage of the 3L-NPC main converter is chosen to be $V_{\mathrm{dc}}=5.3 \mathrm{kV}$. Due to its high voltage and power rating, the 3L-NPC main converter is operated with a space vector modulation (SVM) according to [14] at a low switching frequency of $f_{\mathrm{sw}, \mathrm{mc}}=750 \mathrm{~Hz}$. As a result, the switching frequency per switch position is approximately $325 \mathrm{~Hz}$. The converter-side filter inductance decouples the voltage-source main converter and active filter and limits the main converter current ripple. It is set to $L_{\mathrm{f}, \mathrm{c}}=0.75 \mathrm{mH}$, corresponding to 0.11 per-unit (p.u.). The CHB AF is composed of $n_{\mathrm{af}}=4$ cascaded full-bridge cells per phase with a cell DC-link capacitance of $C_{\mathrm{af}}=0.7 \mathrm{mF}$. The active filter cell voltage is set to $v_{\mathrm{C}_{\mathrm{af}}}=700 \mathrm{~V}$ and the cell carrier frequency is set to $f_{\mathrm{sw} \text {,af }}=8.05 \mathrm{kHz}$. According to [15], during each pulsewidth-modulation (PWM) period a number of cells is permanently turned on and only one cell is switched with a duty cycle and unipolar PWM.

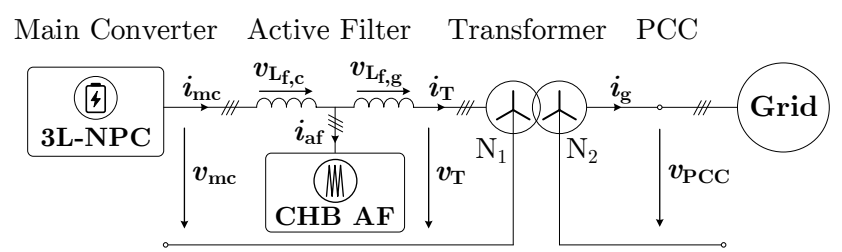

Fig. 5: Proposed grid-connected hybrid medium voltage converter system 
TABLE I: Simulation parameters

\begin{tabular}{|c|c|c|c|c|c|c|c|c|c|c|c|c|}
\hline \multicolumn{4}{|c|}{ System Base Values } & \multicolumn{2}{|c|}{ Transformer } & \multicolumn{3}{|c|}{ 3L-NPC } & \multicolumn{4}{|c|}{ CHB AF } \\
\hline$S_{\mathrm{r}}$ & $V_{\mathrm{r}}$ & $I_{\mathrm{r}}$ & $f_{\mathrm{g}}$ & $v_{\mathrm{k}}$ & $u_{\mathrm{T}, \mathrm{af}}$ & $V_{\mathrm{dc}}$ & $f_{\mathrm{sw}, \mathrm{mc}}$ & $L_{\mathrm{f}, \mathrm{c}}$ & $n_{\mathrm{af}}$ & $v_{\mathrm{C}_{\mathrm{a}}}$ & $C_{\mathrm{af}}$ & $f_{\mathrm{SW}, \mathrm{af}}$ \\
\hline $5 \mathrm{MVA}$ & $3.3 \mathrm{kV}$ & $875 \mathrm{~A}$ & $50 \mathrm{~Hz}$ & $6 \%$ & 0.91 & $5.3 \mathrm{kV}$ & $750 \mathrm{~Hz}$ & $750 \mu \mathrm{H}$ (0.11 p.u.) & 4 & $700 \mathrm{~V}$ & $0.7 \mathrm{mF}$ & $8.05 \mathrm{kHz}$ \\
\hline
\end{tabular}

Fig. 6 shows the voltages and currents of the hybrid converter and the transformer at rated operation. The low switching frequency of the main converter three-level output voltage leads to a main converter current total harmonic distortion (THD) of $9.8 \%$. As a result of the multilevel output voltage and the high effective switching frequency, the CHB AF voltage in good approximation provides a sinusoidally-shaped hybrid converter output voltage. Even for the low output filter inductance given by the transformer leakage inductance, this leads to a nearly sinusoidal output current with a harmonic distortion of only $0.9 \%$ that is injected into the medium voltage grid. Assuming the transformer current as an undistorted fundamental current $\boldsymbol{i}_{\mathrm{T}}={ }^{{ }^{1}} \boldsymbol{i}_{\mathrm{T}}$, the active filter current is given by Kirchhoff's Current Law (KCL) at the converter output

$$
\begin{aligned}
\boldsymbol{i}_{\mathrm{af}}=\boldsymbol{i}_{\mathrm{mc}}-\boldsymbol{i}_{\mathrm{T}} & =\sum_{\nu=2}^{\infty}{ }^{\nu} \boldsymbol{i}_{\mathrm{mc}}+{ }^{\mathbf{1}} \boldsymbol{i}_{\mathrm{mc}}-{ }^{\mathbf{1}} \boldsymbol{i}_{\mathrm{T}} \\
& =\sum_{\nu=2}^{\infty}{ }^{\nu} \boldsymbol{i}_{\mathrm{mc}}+{ }^{\mathbf{1}} \boldsymbol{i}_{\mathrm{af}}
\end{aligned}
$$

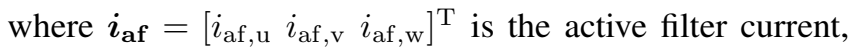
$\boldsymbol{i}_{\mathrm{mc}}=\left[\begin{array}{lll}i_{\mathrm{mc}, \mathrm{u}} & i_{\mathrm{mc}, \mathrm{v}} & i_{\mathrm{mc}, \mathrm{w}}\end{array}\right]^{\mathrm{T}}$ the main converter current, $\boldsymbol{i}_{\mathrm{T}}=\left[\begin{array}{lll}i_{\mathrm{T}, \mathrm{u}} & i_{\mathrm{T}, \mathrm{v}} & i_{\mathrm{T}, \mathrm{w}}\end{array}\right]^{\mathrm{T}}$ the transformer current and $\nu=$ $\{h \in \mathbb{N} \mid h \geq 2\}$ the order of the current harmonics. Due to the very low fundamental current ${ }^{1} \boldsymbol{i}_{\mathrm{AF}}$ required for the CHB AF energy control, the active filter current shown in Fig. 6 corresponds in good approximation to the total main converter current ripple $\sum_{\nu=2}^{\infty}{ }^{\nu} \boldsymbol{i}_{\mathrm{MC}}$ and has a root mean square $(\mathrm{rms})$ value of $I_{\mathrm{af}}=92 \mathrm{~A}$. It is seen that the converter-side filter inductance $L_{\mathrm{f}, \mathrm{c}}$ effectively limits the main converter current ripple to below $300 \mathrm{~A}$, ensuring an operation within the Safe Operating Area (SOA) of the active filter IGBT modules. The main converter and the active filter have an output power of $S_{\mathrm{mc}}=4.46 \mathrm{MVA}$ and $S_{\text {af }}=0.35 \mathrm{MVA}$, respectively. It is worth noting that this leads to an active filter to main converter power rating ratio of only $s=\frac{S_{\mathrm{AF}}}{S_{\mathrm{MC}}}=7.7 \%$ at the investigated operating point, illustrating the advantages of the hybrid converter topology: A conventional main converter with IGBT power modules allows a high power rating of the converter system at low costs, while the parallel multilevel active filter allows a high quality voltage-source output characteristic at only a small share of the total power. Therefore, the multilevel active filter can be designed with low power components at low cost compared to the overall converter system. As a result, the hybrid converter offers high power density and low costs compared to conventional multilevel converters.

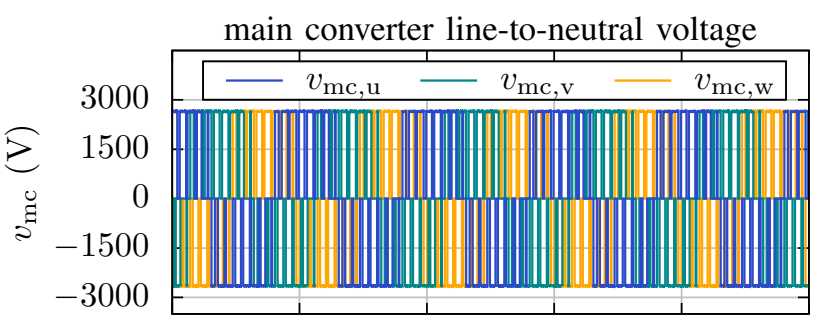

hybrid converter line-to-neutral voltage
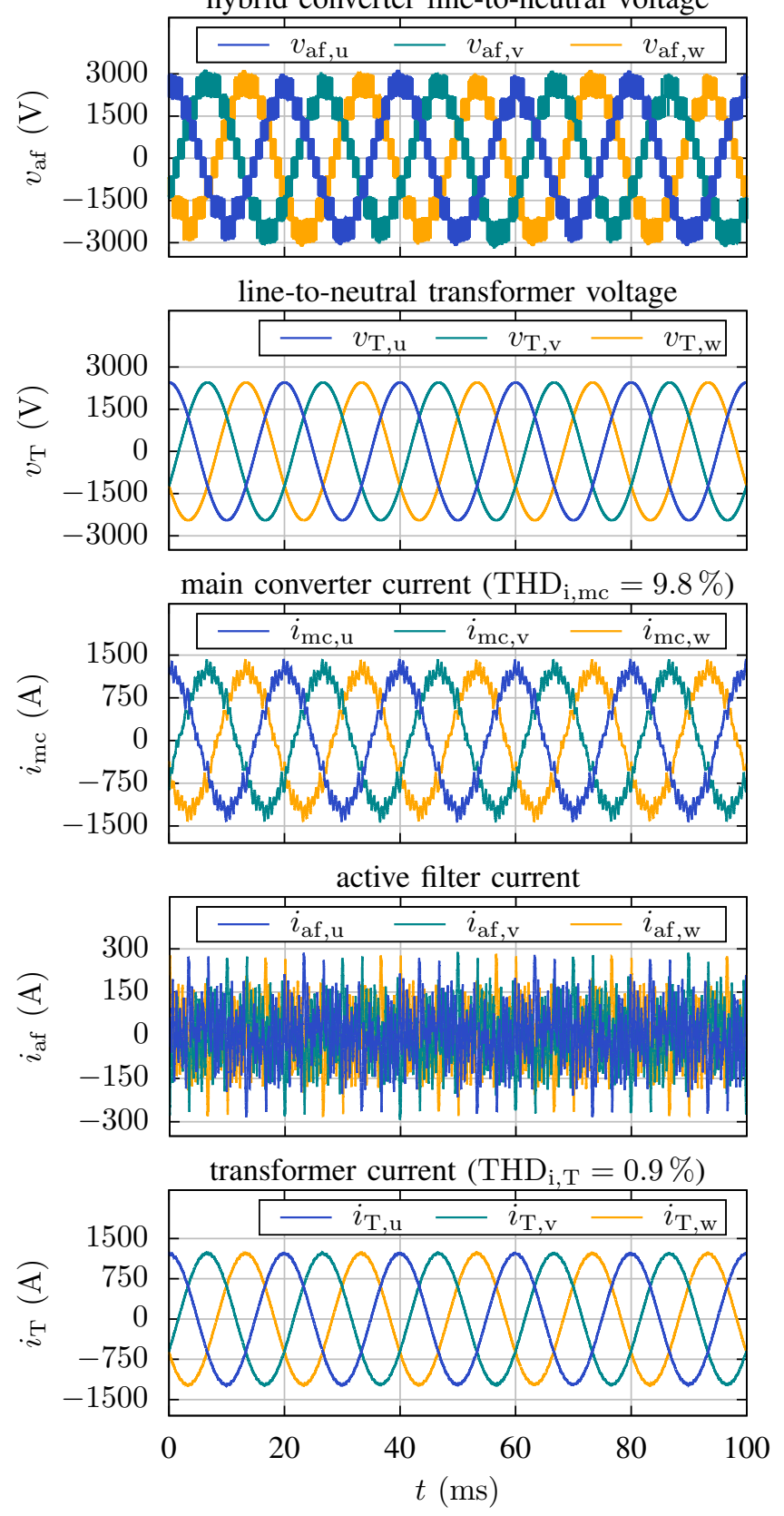

Fig. 6: Simulation results of the hybrid converter system 


\section{CONVERTER Characteristics}

The simulation results in Section III demonstrate the excellent harmonic performance of the hybrid converter system. In order to evaluate the potential for grid applications, converter characteristics of the grid-connected $\mathrm{CHB}$ AF and conventional LCL-filter based converter system are investigated in this section. The fundamental voltage and current loads of the main converter and output filter are derived and the resulting converter utilization and efficiency are presented for the entire power factor range. For readability, the following does not include superscripts to identify fundamental voltages and currents.

\section{A. Hybrid converter system}

The converter analysis is carried out for the hybrid converter system in Fig. 5 and the corresponding parameters in Table I. Inductive and capacitive loads in the power factor range $-1 \leq \cos (\varphi) \leq 1$ with $\varphi=\{\gamma \in \mathbb{R} \mid 0 \leq \gamma \leq 2 \pi\}$ are considered for the operation at rated grid voltage and current.

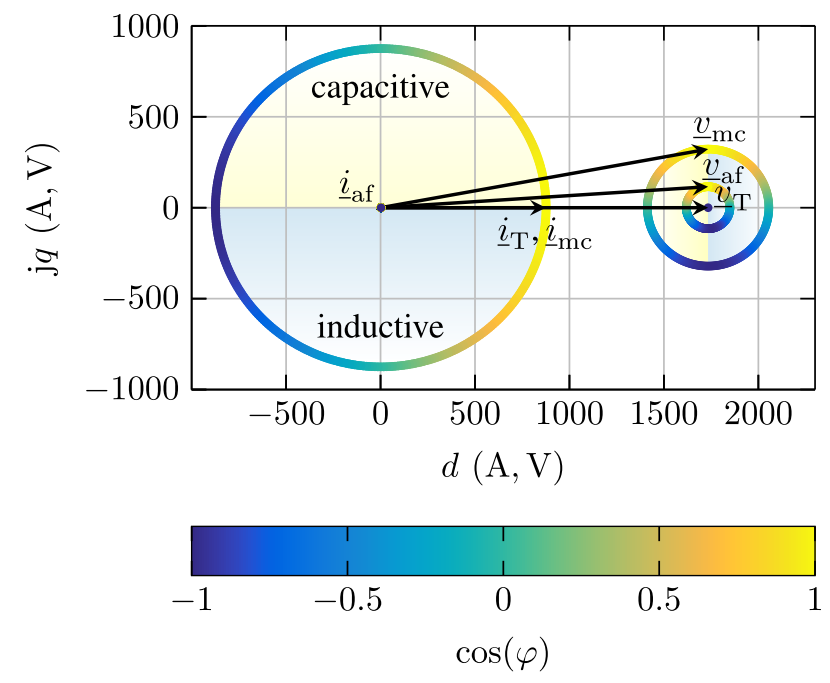

Fig. 7: Complex vector diagram of the hybrid converter system: voltages and currents of the main converter, active filter and transformer

Fig. 7 shows the complex vector diagram of the fundamental voltages and currents in grid synchronous $d q$-coordinates, where all complex vectors corresponding to $\cos (\varphi)=1$ are depicted in black. In relation to a grid-side converter control using a synchronous reference frame phase-locked-loop, the measured transformer primary voltage $\underline{v}_{\mathrm{T}}$ is located on the positive $d$-axis for all load conditions. Hence, the power factor dependent transformer currents $\underline{i}_{\mathrm{T}}(\varphi)$ form a circle around the origin, where inductive and capacitive output currents are located in the lower (blue shading) and the upper (yellow shading) coordinate half plane, respectively.
The voltage drop across the grid-side filter inductance has a $90^{\circ}$ phase shift to $\underline{i}_{\mathrm{T}}(\varphi)$ and is given by

$$
\underline{v}_{\mathrm{L}_{\mathrm{f}, \mathrm{g}}}(\varphi)=j \omega_{\mathrm{g}} L_{\mathrm{f}, \mathrm{g}} \underline{i}_{\mathrm{T}}(\varphi) .
$$

Kirchhoff's Voltage Law (KVL) at the converter output results in the active filter voltage

$$
\underline{v}_{\mathrm{af}}(\varphi)=\underline{v}_{\mathrm{T}}+j \omega_{\mathrm{g}} L_{\mathrm{f}, \mathrm{g}} \underline{i}_{\mathrm{T}}(\varphi) .
$$

The fundamental current $\underline{i}_{\mathrm{af}}(\varphi)$ required for the $\mathrm{CHB} \mathrm{AF}$ energy control is negligible compared to the transformer current. As a result, with respect to KCL at the active filter output, the main converter current $\underline{i}_{\mathrm{mc}}(\varphi)$ corresponds in good approximation to the transformer current:

$$
\underline{i}_{\mathrm{mc}}(\varphi)=\underline{i}_{\mathrm{af}}(\varphi)+\underline{i}_{\mathrm{T}}(\varphi) \approx \underline{i}_{\mathrm{T}}(\varphi)
$$

According to KVL at the main converter output, the active filter voltage and the voltage drop across the converter-side filter inductance yield the main converter voltage

$$
\underline{v}_{\mathrm{mc}}(\varphi)=\underline{v}_{\mathrm{af}}(\varphi)+j \omega_{\mathrm{g}} L_{\mathrm{f}, \mathrm{c}} \underline{i}_{\mathrm{mc}}(\varphi) .
$$

\section{B. Conventional LCL-filter based converter system}

The conventional and commonly used grid-connected converter system with LCL-filter is shown in Fig. 8. Compared to the hybrid converter system in Fig. 5, it replaces the additional power converter with filter capacitors.

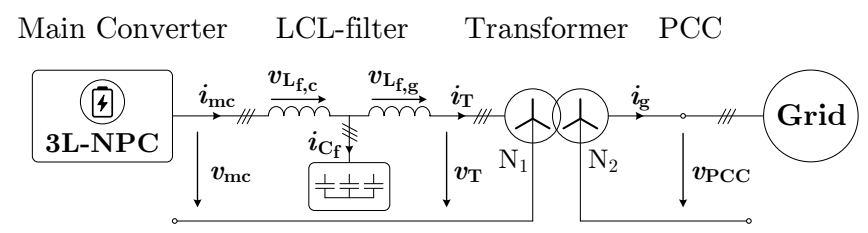

Fig. 8: Conventional grid-connected medium voltage converter system with LCL-filter

The reference LCL-filter parameters used in this work are based on [16] and given in Table II. For the 3LNPC operating parameters given in Table I, the LCL-filter ensures grid code compliance according to the harmonic requirements of IEEE 519. It should be noted that the low switching frequency of the 3L-NPC requires a low LCL-filter resonant-pole frequency corresponding to $5 \mathrm{p}$.u. in order to attain sufficient attenuation. As a consequence, the passive filter components are significantly increased compared to the hybrid converter system: the converter-side and grid-side filter inductance result in $1.25 \mathrm{mH}(0.18$ p.u. $)$ and $1.8 \mathrm{mH}$ (0.26 p.u.), respectively.

To achieve a control reserve corresponding to the hybrid converter system, the resulting transformer turns ratio needs to be reduced compared to the hybrid converter turns ratio in $(5)$ :

$$
u_{\mathrm{T}, \mathrm{LCL}}=\frac{v_{\mathrm{T}}}{v_{\mathrm{PCC}}}=0.8
$$


TABLE II: Reference LCL-filter parameters

\begin{tabular}{lcrr}
\hline Parameter & Symbol & \multicolumn{2}{c}{ Value } \\
\hline Converter-side inductance & $L_{\mathrm{f}, \mathrm{c}(\mathrm{LCL})}$ & 1.25 & $\mathrm{mH}$ \\
Grid-side inductance & $L_{\mathrm{f}, \mathrm{g}(\mathrm{LCL})}$ & 1.8 & $\mathrm{mH}$ \\
Filter capacitance & $C_{\mathrm{f}}$ & 0.55 & $\mathrm{mF}$ \\
Resonant-pole frequency & $\omega_{\mathrm{p}}$ & 1571 & $\mathrm{rad} / \mathrm{s}$ \\
Damping factor & $\zeta_{\mathrm{p}}$ & 0.33 & \\
Damping resistance & $R_{\mathrm{d}}$ & 0.76 & $\Omega$ \\
\hline
\end{tabular}

Furthermore, the oscillation susceptibility of the LCL-filter requires a damping factor of 0.33 , corresponding to a damping resistance of $0.76 \Omega$ and leading to substantial additional losses. According to [16], low-pass RL-damping and resonant RLC-damping circuits can be used to reduce the resulting damping losses. Considering the damping factor and resistance in Table II, an additional damping inductance of $L_{\mathrm{d}, \mathrm{RL}}=1.76 \mathrm{mH}$ is inserted in parallel with the damping resistor for RL-damping. In case of RLCdamping, an additional damping inductance and capacitance of $L_{\mathrm{d}, \mathrm{RLC}}=0.68 \mathrm{mH}$ and $C_{\mathrm{d}, \mathrm{RLC}}=0.6 \mathrm{mF}$ are inserted in parallel with the damping resistor, respectively.

As the LCL-filter transfer function is in good approximation independent of the damping circuit [16], the calculations are performed for R-damping. The resulting fundamental voltages and currents of the LCL-filter based converter system are depicted in the complex vector diagram in Fig. 9. Due to the reduced transformer turns ratio, the transformer primary voltage $\underline{v}_{T}(\varphi)$ is reduced slightly compared to the hybrid converter in Fig. 7. According to (8), the voltage drop across the grid-side filter inductance and the transformer primary voltage result in the LCL-filter voltage $\underline{v}_{\mathrm{C}_{f}}(\varphi)$ across the series-connection of filter capacitors and damping circuit. Hence, the fundamental current through the filter capacitors and damping circuit can be calculated by

$$
\underline{i}_{\mathrm{C}_{\mathrm{f}}}(\varphi)=\frac{\underline{v}_{\mathrm{C}_{\mathrm{f}}}(\varphi)}{\left(j \omega_{\mathrm{g}} C_{\mathrm{f}}\right)^{-1}+R_{\mathrm{d}}} .
$$

As can be seen in Fig. 9, the filter capacitors and damping resistors carry a fundamental current of several hundred amperes in the entire operating range, leading to a significant amount of required capacitive reactive power and substantial additional losses. This clearly shows an important advantage of the hybrid converter system: while the active filter uses DC capacitors and only carries a small fundamental current for semiconductor loss compensation, the AC capacitors of a LCL-filter require a significant amount of reactive fundamental power in order to provide a sinusoidal output voltage of the converter system. Consequently, according to (9), the main converter current $\underline{i}_{\mathrm{mc}}(\varphi)$ clearly differs from the transformer current $\underline{i}_{\mathrm{T}}(\varphi)$ : its absolute value is increased for capacitive and reduced for inductive loads. Considering (10), the voltage drop across the converter-side filter inductance caused by $\underline{i}_{\mathrm{mc}}(\varphi)$ and the LCL-filter voltage $\underline{v}_{\mathrm{C}_{\mathrm{f}}}(\varphi)$ result in a required main converter output voltage $\underline{v}_{\mathrm{mc}}(\varphi)$. Compared to the main converter voltage of the

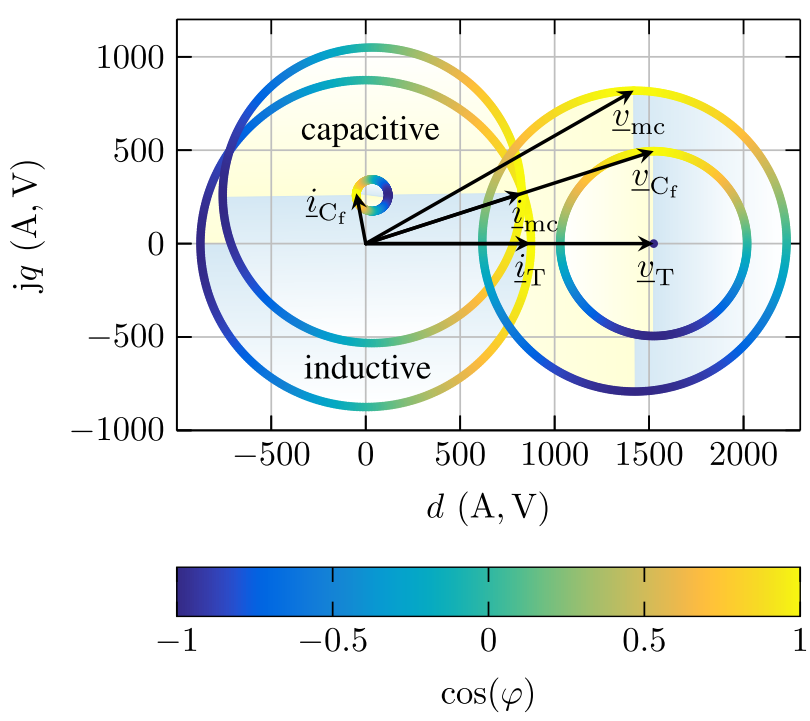

Fig. 9: Complex vector diagram of the LCL-filter based converter system: voltages and currents of the main converter, filter capacitors and transformer

hybrid converter in Fig. 7, the absolute value and angle of $\underline{v}_{\mathrm{mc}}(\varphi)$ have an increased dependency on the load condition. It is seen that the absolute value is significantly increased for inductive and reduced for capacitive loads.

\section{Converter utilization and efficiency}

The previous sections illustrate inherent differences of the hybrid and the LCL-filter based converter system. In this section, the impact of voltage and current characteristics on the achievable converter utilization and efficiency are investigated.

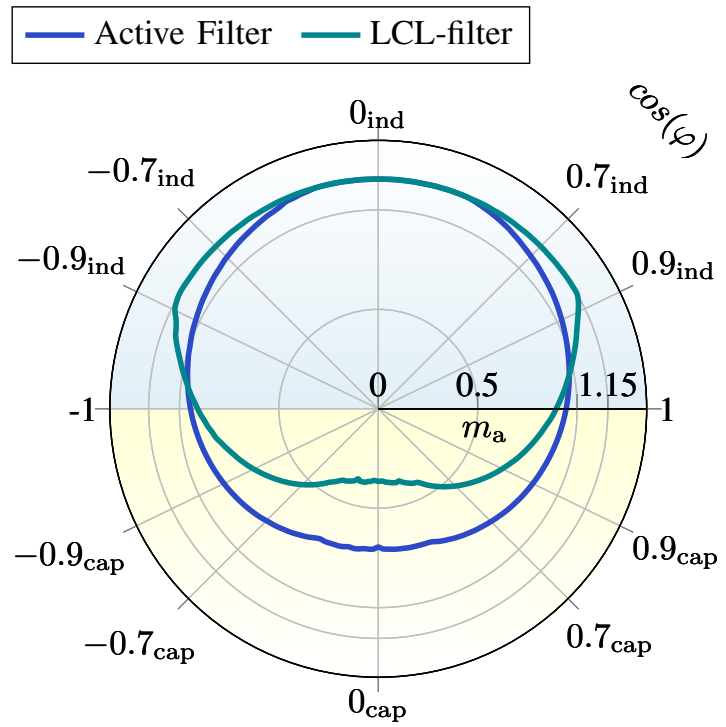

Fig. 10: Main converter modulation-index as a function of the power factor for operation at rated grid voltage and maximum output power 
Taking into account converter limitations such as maximum modulation-index and semiconductor temperatures that must be considered during operation, the LCL-filter complex vector diagram in Fig. 9 reveals characteristics that may lead to a reduced converter utilization. Under capacitive load conditions, the main converter current load is increased compared to the transformer current and can cause an output power limitation due to the maximum junction temperature of the semiconductors. On the other hand, while the main converter current load is reduced for inductive loads, the high main converter voltage can cause an output power limitation due to the maximum modulation-index. In contrast, the load condition dependent variation of the required main converter voltage of the hybrid converter system in Fig. 7 is significantly reduced compared to the conventional converter system. This is clearly seen in Fig. 10, where a large modulation-index range of the conventional converter system is needed for the entire operating range. As expected, for inductive load conditions with power factors of approximately $-0.9_{\text {ind }} \leq \cos (\varphi) \leq 0.9_{\text {ind }}$, the conventional converter system operates with the maximum modulationindex 1.15. The hybrid converter system, however, shows a reduced modulation-index range and is only operating at the modulation-index limit for highly inductive loads with $-0.4_{\text {ind }} \leq \cos (\varphi) \leq 0.4_{\text {ind }}$.

In order to evaluate the achievable converter utilization and efficiency, datasheet-based semiconductor and heat sink models were implemented in the simulation models of the

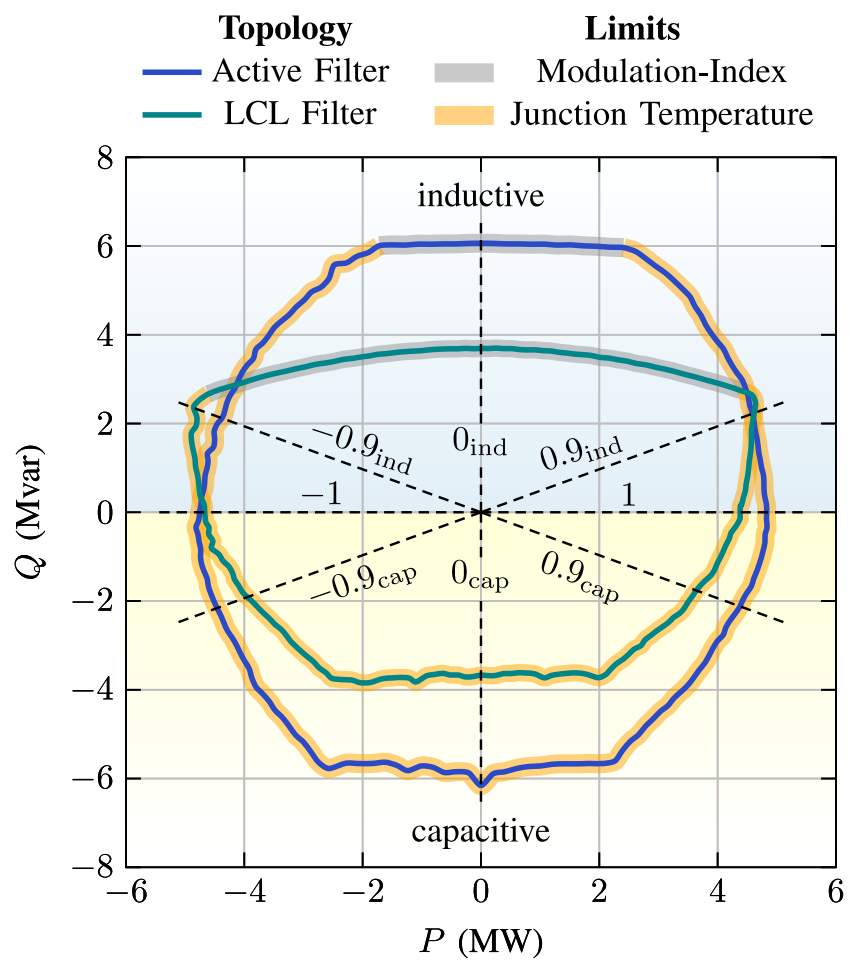

Fig. 11: Maximum output power for all load conditions and a maximum junction temperature of $\vartheta_{\mathrm{j}} \leq 125^{\circ} \mathrm{C}$

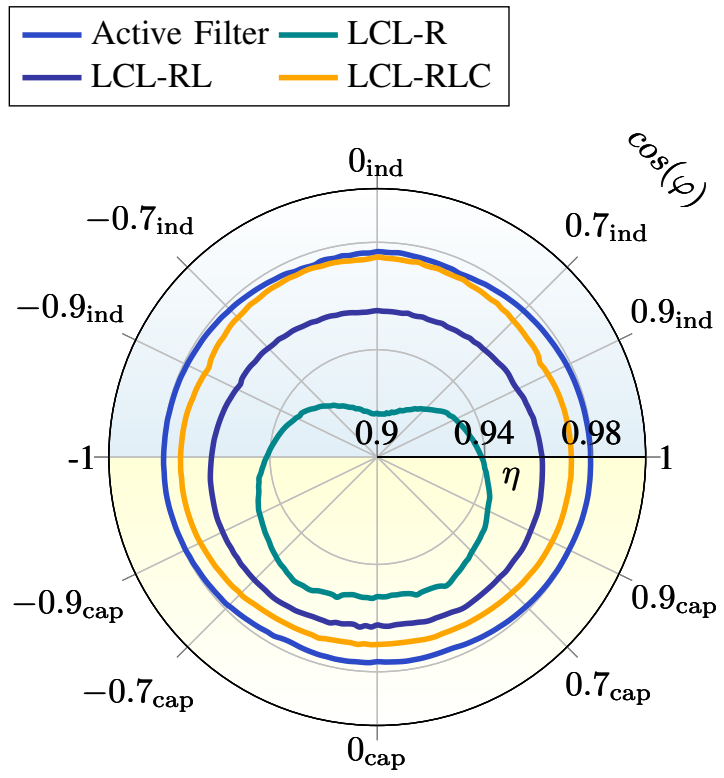

Fig. 12: Efficiency of the hybrid converter and LCL-filter based converter system

converter systems. A maximum junction temperature of $\vartheta_{\mathrm{j}}=125^{\circ} \mathrm{C}$ is assumed for all semiconductors. Fig. 11 shows the maximum reactive and active output power of the converter systems at rated grid voltage with respect to the modulation-index and junction temperature limits. According to Fig. 10, the maximum output power of the LCL-filter based converter system is limited below $4 \mathrm{MVA}$ by the maximum modulation-index for inductive loads. As expected, the increased current load of the main converter under capacitive load conditions causes a significant output power limitation due to the maximum semiconductor junction temperature. In contrast, the homogeneous 3L-NPC current load of the hybrid converter system allows a circular output power characteristic. The output power is limited to approximately $6 \mathrm{MVA}$ only at highly inductive loads due to the maximum modulation-index. However the passive component requirement is significantly reduced compared to the conventional converter system, the hybrid converter system allows a higher output power under almost all load conditions. Since both converter systems use a 3L-NPC with equal operating parameters, this clearly reveals an increased converter utilization with the hybrid converter system.

The corresponding efficiencies of the converter systems are shown in Fig. 12. Therein, main converter losses, filter losses and inductance losses due to an assumed equivalent series resistance of 0.005 p.u. are considered for the loss calculation. As expected, the LCL-filter with resistors as damping circuit (LCL-R) causes significant losses and thereby leads to the lowest converter efficiency. The achievable improvement in efficiency with additional passive components within the damping circuit is seen in Fig. 12: for all load conditions, RLdamping and RLC-damping allow efficiencies above $95 \%$ and $97 \%$, respectively. However, the hybrid converter system 
achieves the highest efficiency over the entire operating range. Significantly increased efficiencies are attained, especially in the commonly used power factor range of $|\cos \varphi| \geq 0.9$.

\section{CONCLUSION}

In this contribution, we propose and investigate a novel hybrid converter topology for medium voltage applications. It is composed of a high power conventional 3L-NPC and a low power Cascaded H-Bridge Converter as active filter. The presented control scheme ensures an accurate energy balancing of the $\mathrm{CHB}$ active filter and leads to excellent harmonic performance under reasonable grid conditions, as shown by the simulation results. In terms of voltage and current load, inherent differences of the hybrid and conventional LCL-filter based converter systems are shown for grid applications: the filter capacitors cause an increased main converter voltage load for inductive and an increased current load for capacitive power factors. In contrast, the active filter of the hybrid converter system leads to approximately homogeneous voltage and current loads in the entire operating range. Even with greatly reduced passive components, this results in a significant increase in maximum output power and a superior converter utilization compared to the conventional converter system. In addition, the hybrid converter clearly surpasses the efficiency of conventional LCL-filter based converter systems. As a result, the proposed hybrid converter constitutes a promising alternative to conventional medium voltage converter solutions and offers significant potential for a wide field of applications.

\section{REFERENCES}

[1] D. Krug, S. Bernet, S. S. Fazel, K. Jalili, and M. Malinowski. "Comparison of 2.3-kV MediumVoltage Multilevel Converters for Industrial MediumVoltage Drives". In: IEEE Transactions on Industrial Electronics 54.6 (Dec. 2007), pp. 2979-2992.

[2] T. Bruckner, S. Bernet, and H. Guldner. "The Active NPC Converter and Its Loss-Balancing Control". In: IEEE Transactions on Industrial Electronics 52.3 (June 2005), pp. 855-868.

[3] T. Bruckner, S. Bernet, and P. Steimer. "The active npc converter for medium-voltage applications". In: Fourtieth IAS Annual Meeting. Conference Record of the 2005 Industry Applications Conference, 2005. IEEE.

[4] IEEE Recommended Practice and Requirements for Harmonic Control in Electric Power Systems.

[5] T. Geyer. "A Comparison of Control and Modulation Schemes for Medium-Voltage Drives: Emerging Predictive Control Concepts Versus PWM-Based Schemes". In: IEEE Transactions on Industry Applications 47.3 (May 2011), pp. 1380-1389.
[6] D. Bernet, L. Stefanski, R. Schwendemann, C. Rollbühler, and M. Hiller. "Grid-Connected Voltage Source Converters with integrated Multilevel-Based Active Filters". In: 2018 IEEE Energy Conversion Congress and Exposition (ECCE). IEEE, Sept. 2018.

[7] C. D. Townsend, T. J. Summers, and R. E. Betz. "Phase-Shifted Carrier Modulation Techniques for Cascaded H-Bridge Multilevel Converters". In: IEEE Transactions on Industrial Electronics 62.11 (Nov. 2015), pp. 6684-6696.

[8] K. Sano and M. Takasaki. "A Transformerless DSTATCOM Based on a Multivoltage Cascade Converter Requiring No DC Sources". In: IEEE Transactions on Power Electronics 27.6 (June 2012), pp. 2783-2795.

[9] E. Behrouzian. "On control of cascaded H-bridge converters for STATCOM applications". PhD thesis. Chalmers University of Technology, 2017.

[10] M. Pereira, D. Retzmann, J. Lottes, M. Wiesinger, and G. Wong. "SVC PLUS: An MMC STATCOM for network and grid access applications". In: 2011 IEEE Trondheim PowerTech. IEEE, June 2011.

[11] ABB AB. SVC Light: For electrical transmission grids. Brochure ABB FACTS, Vasteras, Sweden, 2014.

[12] F. Kammerer, M. Gommeringer, J. Kolb, and M. Braun. "Energy balancing of the Modular Multilevel Matrix Converter based on a new transformed arm power analysis". In: 2014 16th European Conference on Power Electronics and Applications. IEEE, Aug. 2014.

[13] P. Himmelmann and M. Hiller. "A Generalized Approach to the Analysis and Control of Modular Multilevel Converters". In: PCIM Europe 2017; International Exhibition and Conference for Power Electronics, Intelligent Motion, Renewable Energy and Energy Management. May 2017.

[14] N. Celanovic and D. Boroyevich. "A fast spacevector modulation algorithm for multilevel threephase converters". In: IEEE Transactions on Industry Applications 37.2 (2001), pp. 637-641.

[15] F. Kammerer, J. Kolb, and M. Braun. "A novel cascaded vector control scheme for the Modular Multilevel Matrix Converter". In: IECON 2011 - 37th Annual Conference of the IEEE Industrial Electronics Society. IEEE, Nov. 2011.

[16] A. A. Rockhill, M. Liserre, R. Teodorescu, and P. Rodriguez. "Grid-Filter Design for a Multimegawatt Medium-Voltage Voltage-Source Inverter”. In: IEEE Transactions on Industrial Electronics 58.4 (Apr. 2011), pp. 1205-1217. 